\title{
Impacto de la enfermedad cardiovascular en los costos de hospitalización de pacientes con artritis reumatoidea
}

\author{
Ricardo Pineda-Tamayo 1,2, Giovanna Arcila ${ }^{1,3}$, Patricia Restrepo ${ }^{1,3}$, Juan Manuel Anaya ${ }^{1,2}$ \\ ${ }^{1}$ Unidad de Reumatología, Clínica Universitaria Bolivariana, Facultad de Medicina, Universidad Pontificia \\ Bolivariana, Medellín, Colombia. \\ ${ }^{2}$ Unidad de Biología Celular e Inmunogenética, Corporación para Investigaciones Biológicas, Medellín, \\ Colombia. \\ ${ }^{3}$ Facultad de Enfermería, Universidad Pontificia Bolivariana, Medellín, Colombia. \\ El objetivo del presente estudio fue analizar las causas y los costos directos de hospitalización \\ de pacientes con artritis reumatoidea, establecer la morbilidad asociada y evaluar su impacto \\ sobre los costos de la hospitalización. Para tal fin, se revisaron las historias clínicas y los \\ registros del Departamento de Estadística y Contabilidad de todos los pacientes con artritis \\ reumatoidea admitidos a la Clínica Universitaria Bolivariana en Medellín, en el periodo \\ comprendido entre enero de 1999 y junio de 2003. Se hospitalizaron 41 pacientes en 62 \\ oportunidades $(0,34$ hospitalizaciones por paciente por año). La principal causa de \\ hospitalización fue la actividad de la enfermedad $(60 \%)$, seguida de cirugía $(18 \%)$ e infección \\ (10\%). En 30 casos hospitalizados $(48,4 \%)$ se observó, al menos, una morbilidad asociada; la \\ más frecuente fue la enfermedad cardiovascular (32\%). El promedio de estancia fue de $5 \pm 6$ \\ días. El promedio de los costos totales fue de US\$1.277, y el costo promedio del día de \\ hospitalización fue de US\$235. Los medicamentos representaron el $54 \%$ de los costos totales, \\ mientras que los de asistencia médica representaron apenas el 3\%. La enfermedad \\ cardiovascular fue el determinante más importante de altos costos de hospitalización $(p<0,01)$. \\ En conclusión, los costos directos de hospitalización de pacientes con artritis reumatoidea son \\ considerables y surgen principalmente del compromiso orgánico de la enfermedad. La \\ prevención y el tratamiento de la enfermedad cardiovascular son indispensables no sólo para \\ reducir el impacto económico de la artritis reumatoidea, sino también para disminuir el riesgo \\ de mortalidad que la misma acarrea. Estos resultados pueden ser útiles en la definición de las \\ políticas de salud en nuestra población.
}

Palabras clave: artritis reumatoidea, morbilidad asociada, enfermedad cardiovascular, costos directos, hospitalización, infección, mortalidad.

Impact of cardiovascular illness on hospitalization costs in patients with rheumatoid arthritis

The causes of admission and the distribution of direct medical costs were examined to establish the clinical predictors of high hospitalization costs in patients with rheumatoid arthritis. This retrospective study included all rheumatoid arthritis patients who were hospitalized in the Clínica Universitaria Bolivariana in Medellín, Colombia, between January 1999 and June 2003. Data were obtained from the medical records and from the hospital statistical section using a cost-analysis spreadsheet. A total of 41 patients were hospitalized 62 times $(0.34$ hospitalization per patient per year). Disease activity was the most important cause of admission (60\%), followed by surgery $(18 \%)$, and infection $(10 \%)$. In $30(48 \%)$ hospitalizations, at least one comorbidity was recorded, with cardiovascular disease being the most frequent (32\%). The mean length of stay per patient was $5 \pm 6$ days. The mean total cost was US $\$ 1,277$, and the mean cost per day of hospitalization was US $\$ 235$. Medications represented $54 \%$ of the total cost, whereas that representing medical care was only $3 \%$. Variance analysis disclosed cardiovascular disease as the most important determinant of high costs $(p<0.01)$. In conclusion, the direct costs for inpatients with rheumatoid arthritis were considerable, and arose mainly from organic complications. Prevention and treatment of cardiovascular disease are indispensable not only 
to reduce the economic burden of rheumatoid arthitis, but also to diminish the risk of mortality. These data assist in the estimation of health care resources and in the selection of public health policies for the improvement of patient outcomes.

Key words: rheumatoid arthritis, comorbidity, cardiovascular disease, directs costs, hospitalization, infection, mortality.

La artritis reumatoidea es la enfermedad crónica inflamatoria más frecuente ya que afecta, aproximadamente, al $1 \%$ de la población general (1) con una mayor prevalencia en mujeres que en hombres (2). El tratamiento de la artritis reumatoidea precisa el uso de múltiples medicamentos, intervención de diversos especialistas y personal paramédico y, en ocasiones, intervenciones quirúrgicas que generan altos costos a los sistemas de salud, a los pacientes y a sus familias (3). El impacto económico de la artritis reumatoidea es considerable; se ha estimado que los costos por paciente en Estados Unidos oscilan entre US $\$ 4.300$ y US $\$ 5.700$ al año (4). Algunos estudios han revelado que los costos indirectos de la enfermedad pueden ser aún mayores si se considera que muchos pacientes pueden tener una discapacidad laboral en los 10 primeros años de la enfermedad (5). Sin embargo, los costos indirectos se suelen subestimar por ausencia de estudios que los hayan evaluado sistemáticamente (6).

El mayor impacto sobre los costos directos de la artritis reumatoidea está dado por las hospitalizaciones, que representan entre el $40 \%$ y el $60 \%$ del total $(5,7,8)$. Sin embargo, en años recientes, la introducción de la terapia biológica y los elevados costos de ésta han hecho que los costos de hospitalización parezcan menores en términos porcentuales. En tal sentido, un estudio reciente de Michaud et al. (9) evidenció que los costos de hospitalización representaban apenas el $17 \%$ de los costos totales de la artritis reumatoidea.

Los pacientes con artritis reumatoidea tienen mayor morbilidad asociada que la población

\section{Correspondencia}

Ricardo Pineda, Corporación para Investigaciones Biológicas, Carrera 72-A No. 78-B-141, Medellín, Colombia. Teléfono: 441 0855, 441 8846; fax: 4415514 rpineda@cib.org.co

Recibido: 09/07/04; aceptado: 02/09/04 general (10). La hipertensión arterial, la arteriosclerosis, la enfermedad coronaria, los trastornos respiratorios y los cutáneos son morbilidades asociadas frecuentes en la artritis reumatoidea, y tienen un importante impacto en la morbimortalidad; por consiguiente, son responsables de los elevados costos de atención de estos pacientes (10-13).

El propósito del presente estudio fue analizar las causas y los costos directos de hospitalización en pacientes con artritis reumatoidea, establecer la morbilidad asociada y evaluar el impacto de ésta sobre los costos de la hospitalización. Este estudio constituye la segunda fase para el entendimiento de los costos del tratamiento de la artritis reumatoidea en Colombia. Previamente, habíamos reportado los costos directos de la artritis reumatoidea temprana (14).

\section{Materiales y métodos}

\section{Pacientes}

Se revisaron las historias clínicas de todos los pacientes con diagnóstico de artritis reumatoidea admitidos a la Unidad de Reumatología de la Clínica Universitaria Bolivariana (CUB), en el periodo comprendido entre enero de 1999 y junio de 2003. En todos los casos, los pacientes incluidos satisfacían los criterios de clasificación del Colegio Americano de Reumatología (15).

\section{Fuentes de información}

Se utilizaron los datos del Departamento de Estadística y el Departamento de Costos de la CUB utilizando un registro previamente validado para tal fin (coeficiente $\kappa>0,9$ ), en el que se recopiló la información pertinente.

\section{Datos clínicos y demográficos}

Toda la información sobre las características clínicas y demográficas de los pacientes se extractó de la historia clínica y los datos faltantes se obtuvieron por medio de entrevista telefónica, 
personal o ambas con los pacientes. Se estableció la fuente de pago de la siguiente manera:

1. EPS, si el pagador principal era una empresa promotora de salud.

2. Sisbén, si el pagador principal era el régimen de subsidiado. De acuerdo con el Sistema General de Seguridad Social en Colombia, el Sisbén es un sistema para focalizar recursos estatales y de empleados de mayores recursos para la atención a personas que no pueden atender el pago en el régimen de contribución activa al sistema de salud.

3. Particular, si los costos eran asumidos por el paciente o sus familiares.

El estrato socioeconómico se estableció a partir del sistema de estratificación para el pago de servicios públicos en Colombia, entre 1 y 6 , el cual se ha utilizado previamente (16).

\section{Causas de hospitalización y comorbilidad}

Se definió como causa de hospitalización toda condición aguda o crónica que motivara en forma directa la hospitalización. Se incluyeron las siguientes causas: infección, exacerbaciones (actividad de la enfermedad), eventos vasculares (accidente cerebrovascular isquémico o hemorrágico, angina de pecho o infarto de miocardio, isquemia mesentérica), reacción medicamentosa, cirugía de urgencia, cirugía electiva y otras (causas no incluidas en las seis categorías previas).

Se estableció que una condición mórbida asociada era toda aquella patología crónica que acompañaba la artritis reumatoidea que por sí misma podría motivar la hospitalización o no hacerlo. Se evaluó la presencia de las siguientes: hipertensión arterial, enfermedad coronara, diabetes, enfermedad pulmonar crónica, fibromialgia, osteoporosis, anemia, hipotiroidismo y enfermedad cutánea crónica. Las patologías no incluidas en la lista anterior se consideraron como "otras", incluso depresión, enfermedad ácido péptica y síntomas oculares inespecíficos.

\section{Patrón de evaluación de costos}

Previa evaluación de los costos totales a partir de la factura elaborada para el pagador principal al egreso del paciente, se establecieron los costos totales de la admisión y se determinaron, en forma categórica, los siguientes tipos de costos directos:

1. De asistencia médica, representados por todos los costos por evaluaciones de personal médico general y todas las especialidades que hubiesen intervenido en el tratamiento del paciente durante su hospitalización.

2. De la cama, los cuales representaron todos los costos de hotelería, alimentación y cuidados básicos de enfermería.

3. De pruebas de laboratorio, es decir, aquellos costos generados por los perfiles de laboratorio básico no intervencionista, incluidos los estudios de imágenes.

4. De los medicamentos, aquéllos generados por el suministro y la aplicación de medicamentos durante el período de estancia.

5. De procedimientos quirúrgicos.

6. De otros procedimientos diagnósticos, en esta categoría se incluyeron aquellos procedimientos diagnósticos que hubiesen requerido algún tipo de intervencionismo, tales como arteriografías, artrocentesis diagnósticas y terapéuticas, así como biopsias.

7. De material médico-quirúrgico, correspondiente a todos los materiales necesarios para la prestación del servicio médico o quirúrgico, tales como los utilizados en instrumentación quirúrgica, uso de monitores, ventiladores y máquinas de anestesia.

\section{Ajuste de costos}

Los datos se obtuvieron inicialmente en pesos, se ajustaron de acuerdo con el índice de precios al consumidor (IPC) y, posteriormente, se convirtieron a dólares de Estados Unidos a septiembre de 2003.

\section{Análisis estadístico}

Se utilizaron porcentajes y medidas de tendencia central para describir todas las variables clínicas y demográficas, así como para la categorización de los costos generados durante la hospitalización. 
Se realizó un análisis de varianzas entre las diversas variables categóricas y los costos totales. El valor de $p<0,05$ se consideró significativo.

\section{Resultados}

\section{Características clínicas y demográficas}

Durante el período de estudio se hospitalizaron 41 pacientes en 62 oportunidades $(0,34$ hospitalizaciones por paciente por año). La

Cuadro 1. Características clínicas y demográficas de 41 pacientes con artritis reumatoidea que requirieron hospitalización.

\begin{tabular}{lc}
\hline Variable & \\
\hline Edad (años) & $45,2 \pm 14,70$ \\
Mujer (\%) & 71 \\
Duración de la enfermedad (años) & $9,80 \pm 9,01$ \\
Estrato socioeconómico (\%) & \\
1 & 1,6 \\
2 & 32,3 \\
3 & 54,8 \\
4 & 6,5 \\
5 & 4,8 \\
Procedencia (\%) & \\
Rural & 11,3 \\
Urbana & 88,7 \\
Pagador principal (\%) & \\
EPS & 95,2 \\
Particular & 4,8 \\
Motivo de ingreso (\%) & \\
Infección & \\
Actividad de la enfermedad & 9,7 \\
Evento vascular & 59,7 \\
Reacción adversa a medicamentos & 1,6 \\
Cirugía electiva & 1,6 \\
Cirugía de urgencia & 12,9 \\
Otras & 4,8 \\
\hline
\end{tabular}

* Corresponde a 62 hospitalizaciones. principal causa de hospitalización fue la actividad de la enfermedad (cuadro 1). En 30 de las 62 hospitalizaciones $(48,4 \%)$ se observó, al menos, una morbilidad asociada, de las cuales la hipertensión arterial representó el 24,2\% y la enfermedad coronaria el $8,2 \%$. El promedio de estancia fue de $4,95 \pm 6$ días. El cuadro 1 resume otras características clínicas y demográficas de los pacientes que participaron en el estudio.

\section{Análisis de costos}

En $95,2 \%$ de las hospitalizaciones, las EPS fueron los pagadores principales (cuadro 1). Los costos ajustados totales de hospitalización para las 62 hospitalizaciones sumaron US\$79.203. EI promedio de los costos totales fue de US $\$ 1.277$, y el costo promedio del día de hospitalización fue de US\$235.

En el cuadro 2 se muestran los costos por categorías. Los costos de las medicaciones representaron más de la mitad de los costos totales, mientras que los costos de asistencia médica representaron apenas el $3 \%$.

En el análisis de varianza (cuadro 3), el comportamiento de los costos permaneció muy estable con respecto al tiempo de evolución de la artritis reumatoidea y al número de morbilidades asociadas; sin embargo, al analizar éstas en forma individual, se observó un incremento substancial de los costos cuando existía enfermedad coronaria. La presencia de enfermedad cutánea crónica mostró un costo elevado; sin embargo, el incremento desproporcionado de los costos con respecto a esta morbilidad se debió al caso de una sola paciente, cuyos costos totales de

Cuadro 2. Costos directos por tipo, en dólares de Estados Unidos.

\begin{tabular}{lcccr}
\hline Tipo de costo & Suma & Media \pm DE & IC & $\%$ \\
\hline Asistencia médica & $2.392,8$ & $41,2 \pm 66,7$ & $23,7-58,8$ & 3,0 \\
Ocupación de la cama & $10.055,2$ & $176,4 \pm 288,8$ & $99,8-253,0$ & 12,6 \\
Laboratorio & $3.937,4$ & $75,7 \pm 107,6$ & $45,7-105,7$ & 4,9 \\
Medicamentos & $42.706,5$ & $723,8 \pm 2.145,3$ & $164,7-1.282,9$ & 53,9 \\
Procedimientos quirúrgicos & $4.938,4$ & $379,8 \pm 435,9$ & $116,4-643,3$ & 7,2 \\
Otros procedimientos diagnósticos & $1.652,7$ & $38,4 \pm 32,4$ & $28,4-48,4$ & 2,1 \\
Materiales médico-quirúrgicos & $13.265,8$ & $224,8 \pm 766,3$ & $25,2-424,6$ & 16,7 \\
Total & $79.203,6$ & $1277,4 \pm 2.541,2$ & $632,1-1.922,8$ & 100 \\
\hline
\end{tabular}

DE: desviaciones estándar; IC: intervalo de confianza 
Cuadro 3. Análisis de varianza mediante la prueba de Kruskal Wallis para comparaciones entre grupos para los costos totales de 62 hospitalizaciones en 41 pacientes con artritis reumatoidea.

\begin{tabular}{|c|c|c|c|c|}
\hline Variables & $\mathbf{N}$ & $\mathbf{R M}$ & Media $\pm \mathrm{DE}$ & $\mathbf{P}$ \\
\hline \multicolumn{5}{|c|}{ Número de morbilidades asociadas } \\
\hline 0 & 32 & 26,11 & $663,42 \pm 1.150,35$ & 0,09 \\
\hline 1 & 26 & 37,44 & $2.119,44 \pm 3.573,90$ & \\
\hline 2 & 2 & 29,50 & $315,18 \pm 55,93$ & \\
\hline 3 & 2 & 42,50 & $1.119,30 \pm 1.086,26$ & \\
\hline \multicolumn{5}{|l|}{ Morbilidades asociadas } \\
\hline Hipertensión arterial & 15 & 14,80 & $394,94 \pm 381,68$ & 0,004 \\
\hline Enfermedad coronaria & 9 & 31,94 & $3.881,93 \pm 3.173,41$ & \\
\hline Enfermedad cutánea & 7 & 25,43 & $2.516,41 \pm 5.405,41$ & \\
\hline Otras & 10 & 17,35 & $1.375,24 \pm 1.871,58$ & \\
\hline \multicolumn{5}{|l|}{ Días de estancia } \\
\hline $1-2$ & 17 & 16,24 & $245,46 \pm 48,65$ & $<0,001$ \\
\hline $3-7$ & 21 & 29,00 & $1.398,28 \pm 528,91$ & \\
\hline$>8$ & 19 & 40,42 & $2.157,57 \pm 827,10$ & \\
\hline \multicolumn{5}{|c|}{ Duración de la enfermedad (años) } \\
\hline $0-3$ & 16 & 23,78 & $463,42 \pm 815,74$ & 0,09 \\
\hline 4-6 & 14 & 40,00 & $3563,82 \pm 4.486,17$ & \\
\hline $7-15$ & 17 & 29,76 & $694,87 \pm 1.025,48$ & \\
\hline$>16$ & 15 & 33,77 & $672,18 \pm 863,11$ & \\
\hline
\end{tabular}

$\mathrm{RM}$ : rango medio; DE: desviacion estándar

hospitalización fueron de US $\$ 14.770$ y su estancia hospitalaria fue de 44 días.

\section{Discusión}

El impacto económico de las enfermedades reumáticas es enorme. No obstante, aunque su prevalencia e incidencia es alta, la disponibilidad de recursos para atender tales pacientes es limitada $(17,18)$. En el presente estudio se examinaron las causas, los costos y el impacto de la morbilidad asociada en pacientes hospitalizados en un centro universitario de referencia de Medellín, lo que permite obtener datos confiables.

Los resultados señalan que el daño orgánico de la artritis reumatoidea es causa de hospitalización en $60 \%$ de los casos, y que la cirugía y la infección representan el $18 \%$ y el $10 \%$, respectivamente. Es importante señalar que varios pacientes fueron hospitalizados en diversas oportunidades (promedio 1,51 veces), y que el número de hospitalizaciones por paciente por año fue de 0,34. En el presente estudio no se calculó la frecuencia de pacientes con artritis reumatoidea que requirieron hospitalización; no obstante, otros autores han señalado que ésta oscila entre el
$6,5 \%$ y el $15,1 \%(19,20)$. El rango variable de esta frecuencia puede explicarse por la falta de criterios definidos de hospitalización para los pacientes con artritis reumatoidea y por las diferencias en disponibilidad de recursos de cada sistema de salud.

El tiempo promedio de estancia hospitalaria fue de $5 \pm 6$ días y el costo promedio de día hospitalario fue de US\$235. Otros estudios han señalado estancias promedio más prolongadas (19) mientras que algunos autores estiman que el uso de hospitales de día y la atención domiciliaria pudieran ser un recurso para el tratamiento de los pacientes con artritis reumatoidea que requieran hospitalización (20).

En términos porcentuales, el costo de los medicamentos fue el principal rubro seguido por el costo del material médico-quirúrgico (utilizado en monitoría, asistencia y procedimientos), mientras que los costos de los honorarios médicos representaron sólo 3\%. El número de morbilidades asociadas per se no mostró tener impacto significativo sobre los costos. Por el contrario, la enfermedad cardiovascular fue un determinante importante en los costos de la hospitalización. 
De manera similar, pero no sorprendente, el número de días de estancia fue proporcional al incremento de los costos (cuadro 3).

Es frecuente que los pacientes con artritis reumatoidea tengan condiciones asociadas que pueden incrementar su morbimortalidad. Kroot et al. (21) mostraron que el $27 \%$ de los pacientes con artritis reumatoidea tuvieron, al menos, una comorbilidad y que la hipertensión arterial y la angina de pecho fueron las más frecuentes. En el mismo estudio, las enfermedades cardiovasculares fueron las responsables del $29 \%$ de toda la morbilidad asociada (21). En el presente estudio se registró hipertensión arterial en el $24 \%$ de las hospitalizaciones (cuadro 3). Cifra muy similar (21\%) se registró en 168 pacientes ambulatorios tratados en nuestra Unidad (22).

La enfermedad cardiovascular es una causa importante de mortalidad en los pacientes con artritis reumatoidea (23-25). A pesar de que el tabaquismo ha sido implicado como un factor de susceptibilidad y gravedad de la artritis reumatoidea (26-28), no se asocia significativamente con la morbilidad asociada ni con el riesgo de enfermedad cardiovascular en estos pacientes $(22,27,29)$. Si bien algunos factores de riesgo tradicionales podrían estar implicados en el desarrollo de la enfermedad cardiovascular en la artritis reumatoidea (23-26), es la inflamación propia de la enfermedad el principal factor de disfunción endotelial y génesis de arteriosclerosis (12,31-33). Se han estudiado varios marcadores en la enfermedad cardiovascular asociada con la artritis reumatoidea, entre los cuales están la velocidad de sedimentación globular y la proteína C reactiva, así como niveles elevados de anticuerpos contra lipoproteínas de baja densidad oxidadas, aumento en la expresión de moléculas de adhesión endotelial, niveles elevados de homocisteína, algunos marcadores de trombosis como el fibrinógeno, alteración de la fibrinólisis y otros estados de hipercoagulabilidad (31-34).

El evento inicial en el desarrollo de la enfermedad cardiovascular en la artritis reumatoidea es la disfunción endotelial cuyas vías efectoras pueden estar dirigidas desde la misma articulación a través de la producción elevada de citocinas como el FNT $\alpha$ e IL-6 que tienen la capacidad de interferir en diferentes niveles del metabolismo (35), ya que producen alteraciones en el perfil lipídico y en la resistencia periférica a la insulina, promueven la expresión de moléculas de adhesión, incrementan el estrés oxidativo, estimulan la síntesis de óxido nítrico por parte de los monocitos y las células endoteliales, e incrementan la oxidación de las LDL, todo lo cual acelera el proceso de aterogénesis (36).

En el presente estudio las infecciones motivaron la hospitalización en cerca del $10 \%$ de los casos (cuadro 1). Éstas pueden ser causa de morbilidad y mortalidad en los pacientes con artritis reumatoidea (12). No obstante, es difícil diferenciar entre el peso que tiene la enfermedad en sí misma y el efecto del tratamiento de la enfermedad en el desarrollo de las mismas. Por un lado, los pacientes con artritis reumatoidea tienen alteraciones en la inmunidad celular que incluyen disminución en el número y la función de los linfocitos T supresores y de las células NK y, por otro, es conocido el efecto inmunosupresor de los fármacos, tales como los esteroides y el metotrexato (37). La incidencia de infecciones en pacientes con artritis reumatoidea se ha reportado de 0,17 nuevas infecciones por paciente-año, principalmente del tracto respiratorio superior, la piel y el tracto urinario, y favorecidas por el metotrexato y los esteroides (38). La reciente aparición de medicamentos biológicos bloqueadores del FNT $\alpha$, en especial el Infliximab, se ha asociado al desarrollo de tuberculosis (39).

Otras morbilidades asociadas como la enfermedad ácido péptica y la osteoporosis no tuvieron un gran impacto sobre los costos directos de hospitalización en el presente estudio; no obstante, ambas deben ser evaluadas en los pacientes con artritis reumatoidea, dado que tienen impacto, no sólo en la calidad de vida de los mismos, sino también sobre los costos generados por la enfermedad $(11,12)$.

En resumen, el presente estudio evaluó las causas y los costos de la hospitalización de los pacientes con artritis reumatoidea, señalando la enfermedad cardiovascular como la principal morbilidad asociada, con un significativo impacto en el 
incremento de los costos directos de ésta. Por lo tanto, su manejo eficaz así como la prevención y el control de la hipertensión arterial, deben ser considerados en el tratamiento de los pacientes con artritis reumatoidea, no sólo para reducir los costos que ella genera sino también para prevenir el riesgo de mortalidad que la enfermedad cardiovascular acarrea $(23-25,40)$. A la luz de estos resultados consideramos pertinente mencionar algunas estrategias a fin de mejorar el estado funcional e impacto económico de esta enfermedad:

1. Intervención temprana y adecuada. La terapia con medicamentos modificadoras de la enfermedad debe iniciarse tan temprano como sea posible dentro de los tres primeros meses de inicio de los síntomas con el fin de buscar la remisión de la enfermedad y disminuir la posibilidad de aparición de erosiones y deformidades articulares. Cuando la respuesta a las dosis adecuadas de metotrexato es subóptima, debe considerarse la combinación de varios fármacos modificadores de la enfermedad o el uso de terapia biológica con anti-FNT $\alpha$ (41-43). La intervención temprana y la terapia combinada con fármacos modificadores de la enfermedad han mostrado ser más eficaces en el control de la enfermedad y en la reducción de los costos en comparación con el uso de terapia única $(14,45)$. El tratamiento antiinflamatorio eficaz puede evitar el desarrollo de arterioesclerosis y enfermedad cardiovascular y, por tanto, disminuir la mortalidad y el elevado costo de la artritis reumatoidea $(32,45)$.

2. Hospitales de día. Diversas formas de atención de los pacientes durante el día, así como otros mecanismos de cuidado ambulatorio por personal de enfermería cualificado ha demostrado ser una estrategia efectiva en el tratamiento de la artritis reumatoidea que amerita su pronta implantación y regulación por parte de los actores del sistema de salud (46-48).

3. Consideración y reducción de los costos indirectos. Los costos indirectos de una enfermedad están representados por la pérdida de productividad. Desde la perspectiva social, en la que se evalúan todos los costos generados por la enfermedad, tanto para pagadores principales como para el aparato productivo de una nación, los costos indirectos han sido subestimados en la mayoría de los estudios económicos; no obstante, se ha mencionado que éstos pueden superar entre dos y tres veces el valor de los costos directos $(49,50)$. Por lo tanto, es necesario limitar las ausencias laborales y las jubilaciones prematuras por medio de intervenciones tempranas y adecuadas en el curso de la artritis reumatoidea, tal como se mencionó anteriormente.

\section{Agradecimientos}

Los autores expresan su gratitud a los tres revisores anónimos del presente trabajo por sus importantes opiniones; a José Humberto Duque por su apoyo al desarrollo del presente trabajo, a Gabriel J. Tobón y José F. Camargo por su colaboración en la consulta de pacientes, y a Ángela Restrepo por su lectura crítica del manuscrito.

\section{Referencias}

1. Lawrence RC, Helmick CG, Arnett FC, Deyo RA, Felson DT, Giannini EH et al. Estimates of the prevalence of arthritis and selected musculoskeletal disorders in the United States. Arthritis Rheum 1998;41: 778-99.

2. Hochberg MC, Spector TD. The epidemiology of rheumatoid arthritis: an update. Epidemiol Rev 1990; 12:247-52.

3. Griffith J, Carr A. What is the impact of early rheumatoid arthritis on the individual? Baillieres Best Pract Res Clin Rheumatol 2001;15:77-90.

4. Yelin EH. The cost of rheumatoid arthritis: absolute, incremental, and marginal estimates. J Rheumatol 1996; 44(Suppl.):47-51.

5. Callahan LF. The burden of rheumatoid arthritis: facts and figures. J Rheumatol 1998;25(Suppl):8-12.

6. Weisman MH, Gano AD Jr, Gabriel SE, Hochberg MC, Kavanaugh A, Liang MH et al. On behalf of the evidence-based medicine working groups in rheumatology. Reading and interpreting economic evaluations in rheumatoid arthritis: an assessment of selected instruments for critical appraisal. J Rheumatol 2003;30:1339-47.

7. Pugner KM, Scott DI, Holmes JW, Hieke K. The costs of rheumatoid arthritis: an international long-term view. Semin Arthritis Rheum 2000;29:305-20. 
8. Yelin E, Wanke LA. An assessment of the annual and long-term direct costs of rheumatoid arthritis: the impact of poor function and functional decline. Arthirits Rheum 1999;42:1209-18.

9. Michaud K, Messer J, Choi HK, Wolfe F. Direct medical costs and their predictors in patients with rheumatoid arthritis: a three-year study of 7,527 patients. Arthirits Rheum 2003;48:2750-62.

10. Gabriel SE, Crowson CS, O'Fallon WM. Comorbidity in arthritis. J Rheumatol 1999;26:2475-9.

11. Rupp I, Boshuizen HC, Jacobi CE, Dinant HJ, van den Bos G. Comorbidity in patients with rheumatoid arthritis: effect on health-related quality of life. J Rheumatol 2004;31:58-65.

12. Mikuls TR, Saag KG. Comorbidity in rheumatoid arthritis. Rheum Dis Clin North Am 2001;27:283-303.

13. Kroot EJ, van Gestel AM, Swinkels HL, Albers MM, van de Putte LB, van Riel PL. Chronic comorbidity in patients with early rheumatoid arthritis: a descriptive study. J Rheumatol 2001;28:1511-7.

14. Pineda-Tamayo R, Arcila G, Restrepo P, Tobón GJ, Camargo JF, Anaya JM. Costos médicos directos de la artritis reumatoide temprana. Rev Colomb Reumatol 2004;11:89-96.

15. Arnett FC, Edworthy SM, Bloch DA, McShane DJ, Fries JF, Cooper NS et al. The American Rheumatism Association 1987 revised criteria for the classification of rheumatoid arthritis. Arthritis Rheum 1988;31:31524.

16. Cadena J, Vinaccia S, Pérez A, Rico MI, Hinojosa R, Anaya JM. The impact of disease activity on quality of life and mental health status in Colombian patients with rheumatoid arthritis. J Clin Rheumatol 2003;9:142-50.

17. Yelin EH, Callahan LF. The economic cost and social psychological impact of musculoeskeletal conditions. Arthritis Rheum 1995;38:1351-62.

18. Lubeck DP. A review of the direct costs of rheumatoid arthritis: managed care versus fee-for-service settings. Pharmacoeconomics 2001;19:811-8.

19. Wolfe F, Kleinheksel SM, Spitz PW, Lubeck DP, Fries JF, Young DY et al. A multicenter study of hospitalization in rheumatoid arthritis. Frequency, medical-surgical admissions, and charges. Arthritis Rheum 1986;29:614-9.

20. Jacobs J, Keyserling JA, Britton M, Morgan GJ Jr, Wilkenfeld $\mathbf{J}$, Hutchings $\mathrm{HC}$. The total cost of care and the use of pharmaceuticals in the management of rheumatoid arthritis: the Medi-Cal program. J Clin Epidemiol 1988;41:215-23.

21. Kroot EJ, van Gestel AM, Swinkels HL, Albers MM, van de Putte LB, van Riel PL. Chronic comorbidity in patients with early rheumatoid arthritis: a descriptive study. J Rheumatol 2001;28:1511-7.
22. Díaz LA, Tobón GJ, Cadena J, Camargo JF, Pineda R, Anaya JM. Comorbidity in patients with rheumatoid arthritis. Ann Rheum Dis 2004;63(Suppl.1):192.

23. Jacobsson LT, Knowler WC, Pillemer S, Hanson RL, Pettitt DJ, Nelson RG et al. Rheumatoid arthritis and mortality. A longitudinal study in Pima Indians. Arthritis Rheum 1993;36:1045-53.

24. Symmons DP, Jones MA, Scott DL, Prior P. Longterm mortality outcome in patients with rheumatoid arthritis: early presenters continue to do well. J Rheumatol 1998; 25:1072-7.

25. Kvalvik AG, Jones MA, Symmons DPM. Mortality in a cohort of Norwegian patients with rheumatoid arthritis followed from 1977 to 1992 . Scandinavian J Rheumatol 2000;29:29-37.

26. Heliovaara M, Aho K, Aromaa A, Knekt P, Reunanen A. Smoking and risk of rheumatoid arthritis. J Rheumatol 1993;20:1830-5.

27. Silman AJ, Newman J, MacGregor AJ. Cigarette smoking increases the risk of rheumatoid arthritis: results from a nationwide study of disease discordant twins. Arthritis Rheum 1996,39:732-5.

28. Wolfe F. The effect of smoking on clinical, laboratory, and radiographic status in rheumatoid arthritis. J Rheumatol 2000;27:630-7.

29. Wallberg-Jonsson S, Johansson H, RantapääDahlqvist S. Extent of inflammation predicts cardiovascular disease and overall mortality in seropositive rheumatoid arthritis. A retrospective cohort study from disease onset. J Rheumatol 1999,26:256271.

30. Del Rincon ID, Williams K, Stern MP, Freeman GL, Escalante A. High incidence of cardiovascular events in a rheumatoid arthritis cohort not explained by traditional cardiac risk factors. Arthritis Rheum 2001; 44:2737-45.

31. Goodson N. Coronary artery disease and rheumatoid arthritis. Curr Opin Rheumatol 2002;14:115-20.

32. Wallberg-Jonsson S, Cvetkovic JT, Sundqvist KG, Lefvert AK, Rantapaa-Dahlqvist S. Activation of the immune system and inflammatory activity in relation to markers of atherothrombotic disease and atherosclerosis in rheumatoid arthritis. J Rheumatol 2002;29:875-82.

33. Kullo I, Gau G, Tajik A. Novel risk factors for atherosclerosis. Mayo Clin Proc 2000;75:369-80.

34. Ridker PM, Manson JE, Buring JE, Shih J, Matias M, Hennekens $\mathbf{C H}$. Homocysteine and risk of cardiovascular disease among post menopausal women. JAMA 1999;28:1817-21.

35. Camargo JF, Correa PA, Vélez S, Anaya JM. Citoquinas en artritis reumatoide. En: Ramirez LA, Anaya JM, editores. Artritis reumatoide. Medellín, Colombia: Editora Médica Edimeco S.A.; 2004. p.53-80. 
36. Sattar N, McCarey DW, Capell H, Mclnnes IB. Explaining how "high-grade" systemic inflammation accelerates vascular risk in rheumatoid arthritis. Circulation 2003;24:2957-63.

37. Boerbooms AM, Kerstens PJ, van Loenhout JW, Mulder J, van de Putte LB. Infections during low-dose methotrexate treatment in rheumatoid arthritis. Semin Arthritis Rheum 1995;24:411-21.

38. Hernandez-Cruz B, Cardiel MH, Villa AR, AlcocerVarela J. Development, recurrence, and severity of infections in Mexican patients with rheumatoid arthritis. A nested case-control study. J Rheumatol 1998;25: 1900-7.

39. Kroesen S, Widmer AF, Tyndall A, Hasler P. Serious bacterial infections in patients with rheumatoid arthritis under anti-TNF-alpha therapy. Rheumatology (Oxford) 2003;42:617-21.

40. Bacon PA, Townend JN. Nails in the coffin: increasing evidence for the role of rheumatic disease in the cardiovascular mortality of rheumatoid arthritis. Arthritis Rheum 2001;44:2707-10.

41. O'Dell J. Therapeutic strategies for rheumatoid arthritis. N Engl J Med 2004;350:2591-602.

42. Olsen NJ, Stein CM. Drug therapy: new drugs for rheumatoid arthritis. N Engl J Med 2004;350:2167-79.

43. Weinblatt ME, Kremer JM, Bankhurst AD, Bulpitt KJ, Fleischmann RM, Fox RI et al. A trial of etanercept, a recombinant tumor necrosis factor receptor: Fc fusion protein, in patients with rheumatoid arthritis receiving methotrexate. N Engl J Med 1999; 340:253-59.

44. Puolakka K, Kautiainen H, Mottonen T, Hannonen $\mathbf{P}$, Korpela $\mathbf{M}$, Julkunen $\mathbf{H}$ et al. Impact of initial aggressive drug treatment with a combination of disease-modifying antirheumatic drugs on the development of work disability in early rheumatoid arthritis: a five-year randomized follow-up trial. Arthritis Rheum 2004;50:55-62.

45. Minaur NJ, Jacoby RK, Cosh JA, Taylor G, Rasker JJ. Outcome after 40 years with rheumatoid erthritis: a prospective study of function, disease activity and mortality. J Rheumatol 2004;31(Suppl.69):3-8.

46. Tijhuis GJ, Zwinderman AH, Hazes JM, Van Den Hout WB, Breedveld FC, Vliet Vlieland TP. A randomized comparison of care provided by a clinical nurse specialist, an inpatient team, and a day patient team in rheumatoid arthritis. Arthritis Rheum 2002;47: 525-31.

47. Lambert CM, Hurst NP, Forbes JF, Lochhead A, Macleod M, Nuki G. Is day care equivalent to inpatient care for active rheumatoid arthritis? Randomized controlled clinical and economic evaluation. BMJ 1998; 316:965-9.

48. van den Hout WB, Tijhuis GJ, Hazes JM, Breedveld FC, Vliet Vlieland TP. Cost effectiveness and cost utility analysis of multidisciplinary care in patients with rheumatoid arthritis: a randomized comparison of clinical nurse specialist care, inpatient team care, and day patient team care. Ann Rheum Dis 2003;62:308-15.

49. Kobelt G, Eberhardt K, Jönsson L, Jönsson B. Economic consequences of the progression of rheumatoid arthritis in Sweden. Arthritis Rheum 1999; 42:347-56.

50. Lajas C, Abasolo L, Bellajdel B, Hernández-García C, Carmona L, Vargas E et al. Costs and predictors of costs in rheumatoid arthritis: a prevalence-based study. Arthritis Care Res 2003;49:64-70. 